Multilevel airway obstruction including rare tongue base mass presenting as severe croup in an infant

$$
\begin{gathered}
\text { Tara Brennan, } \text { MD }^{2,3} \\
\text { Jeffrey C. Rastatter, MD, FAAP }{ }^{1,2}
\end{gathered}
$$

${ }^{1}$ Department of Otolaryngology, Northwestern University Feinberg School of Medicine, Chicago, IL

${ }^{2}$ Division of Pediatric Otolaryngology, Children’s Memorial Hospital, Chicago, IL

${ }^{3}$ Department of Otolaryngology, University of Illinois at Chicago College of Medicine, Chicago, IL

Corresponding author: Jeffrey C. Rastatter, MD, Division of Pediatric Otolaryngology, Children's Memorial Hospital, 2300 Children’s Plaza, Box \#25, Chicago, IL 60614. Phone: 773.880.4457.

Email address: jrastatter@childrensmemorial.org

Key Words: pediatric upper airway obstruction, laryngomalacia, lingual thyroglossal duct cyst, supraglottoplasty, synchronous airway lesions

Abbreviations: GER (gastroesophageal reflux), SGS (subglottic stenosis), DML (direct microscopic laryngoscopy), FFL (flexible fiberoptic laryngoscopy)

Word count: 1015

Financial disclosures: none

Conflict of interest: none 


\begin{abstract}
:
Laryngomalacia is the most common cause of neonatal stridor, accounting for up to $60 \%$ of cases $^{1}$. Less common causes of neonatal stridor include subglottic or tracheal stenosis, or congenital masses of the upper airway. Neonates with an identified congenital etiology of symptomatic upper airway obstruction often have synchronous airway lesions leading to multilevel airway obstruction. These infants deserve an endoscopic evaluation to better diagnose and manage respiratory distress. Here we present a rare case of an infant initially diagnosed with croup, but ultimately found to have multilevel airway obstruction including severe laryngomalacia and an obstructing tongue base mass.
\end{abstract}

Keywords: pediatric upper airway obstruction, laryngomalacia, lingual thyroglossal duct cyst, supraglottoplasty, synchronous airway lesions 
Introduction:

Laryngotracheobronchitis, or "croup”, is a viral, self-limited illness characterized by a barking cough, inspiratory stridor, and varying degree of respiratory distress. Croup affects about 15\% of children, and typically occurs between the ages of six months and five years ${ }^{2}$.

Laryngomalacia is the commonest cause of neonatal stridor cited in the literature, accounting for up to $60 \%$ of cases $^{1}$. While nearly $90 \%$ will resolve spontaneously, approximately $10 \%$ of patients have severe laryngomalacia that may require surgical intervention ${ }^{3,4}$. Severe laryngomalacia is characterized by respiratory distress, cyanosis, and failure to thrive. Severe laryngomalacia has been shown to coexist with synchronous airway lesions in up to $60 \%$ of cases $^{\mathrm{i}}$. It also often coexists with, and is exacerbated by, gastroesophageal reflux ${ }^{5}$.

Vallecular cysts and tongue base lesions are rare in infants, but when present can cause airway obstruction. Cases of concomitant laryngomalacia and vallecular cysts have been reported in the pediatric literature, albeit uncommonly ${ }^{6,7}$. Yao et $\mathrm{al}^{5}$ found fewer than five such cases in the literature.

We present a case of an infant initially thought to have severe croup, but later found to have multi-level airway obstruction including the rare association of a tongue base mass and laryngomalacia. 


\section{Patient Presentation:}

Here we present a six week old term male who presented to an outside hospital for stridor and failure to thrive. He was treated for croup with racemic epinephrine and nebulized and systemic steroids for six days without significant improvement in symptoms, and subsequently transferred to Children’s Memorial Hospital for further evaluation and treatment. There, his respiratory therapy was continued in the ICU, where he was also initiated on anti-reflux medication. Early in his inpatient stay, he suffered an acute and prolonged apneic event for which a code was called. The patient recovered and was not intubated. At the time, it was thought that the event may have been caused by reflux related aspiration or laryngospasm in the setting of acute laryngotracheobronchitis. The Pediatric Otolaryngology service was consulted.

On flexible fiberoptic laryngoscopy (FFL), the patient was found to have what appeared to be a vallecular or tongue base lesion. Direct microscopic laryngoscopy (DML) in the operating room revealed a large, right-sided vallecular and tongue base mass. The appearance was reminiscent of thyroid tissue. Needle aspiration demonstrated serous fluid. Biopsy was performed and results were consistent with squamous lined cyst. No thyroid tissue was identified on frozen section analysis, but the gross appearance was most consistent with a lingual thyroglossal duct cyst. At that time, the decision was made to transfer the patient, intubated, to the ICU, with plans to obtain a postoperative MRI. This imaging revealed resolution of the cystic component, but persistent $1.3 \times 0.9 \times 1.0 \mathrm{~cm}$ diffusely enhancing, T2 hyperintense lesionin the right tongue 
base. Importantly, his thyroid gland was present and in the normal location and thyroid function tests (TFTs) were within normal limits.

Postoperatively, the patient's stridor and oral feeding intolerance persisted. At one week postop, a repeat bedside FFL was performed. The tongue base lesion was no longer obstructing the airway. However, severe laryngomalacia and laryngeal edema was appreciated (see Video 1). The patient was then taken back to the operating room for direct laryngoscopy, bronchoscopy, and supraglottoplasty. Supraglottoplasty was performed using cold technique. Bronchoscopy at the time revealed $50 \%$ subglottic stenosis.

Postoperatively, the patient's stridor resolved and feeding was improved. He required temporary nasogastric tube feedings due to mild aspiration. The nasogastric tube was removed 3 weeks postoperatively, after which he tolerated oral diet and gained weight. Six months later, the patient continues to gain weight on a regular diet, and has no feeding difficulty or signs of aspiration. Patient continues to follow up with the Otolaryngology and Speech specialists, and has been maintained on anti-reflux medication. Importantly, patient no longer has difficulty breathing or stridor.

\section{Discussion:}

This patient had multiple sites of upper airway obstruction contributing to his difficulty feeding and respiratory distress. These were appreciated only after endoscopic work up and 
intervention. The presence of his large tongue base cyst prevented visualization and diagnosis of the severe laryngomalacia on initial evaluation by FFL. The contribution of laryngomalacia to the airway obstruction became apparent on bedside FFL done after decompression of the tongue base cyst. The larygomalacia was certainly present to some degree independent of the tongue mass as evidenced by the congenitally short aryepiglottic folds and large cuneiform cartilages. One can speculate, however, that the tongue mass may have contributed to the development of severe laryngomalacia through progressive pressure on the delicate epiglottis. The posterior GER likely plays both a cause and effect role: increasing edema in the larynx contributes to bulk in the glottis and supraglottic airway, leading to increased upper airway obstruction, which, in turn, increases negative pressure in the larynx, leading to further supraglottic collapse and reflux. His synchronous subglottic stenosis (SGS) and tracheobronchomalacia also contribute to the upper airway obstruction. SGS specifically can cause "croup like” symptoms and could have contributed to the initial incorrect diagnosis of acute croup infection. This case warrants discussion as it reminds the clinician to consider multiple potential sites of airway obstruction in a neonate with stridor in acute or recurrent respiratory distress, as well as the importance of endoscopic evaluation. Similar to the commonly repeated saying "everything that wheezes is not asthma," it is equally true that "everything that squeaks is not croup." 
References:

1) Holinger, LD. Etiology of stridor in the neonate, infant, and child. AnnOtolRhinol Laryngol 1980;89:397-400.

2) “Diagnosis and Management of Croup” (PDF). BC Children's Hospital Division of Pediatric Emergency Medicine Clinical Practice Guidelines.

3) Holinger LD, Konior RJ. Surgical management of severe laryngomalacia. Laryngoscope 1989;99:136-142.

4) Schroeder J. Synchronous Airway Lesions and Outcomes in Infants With Severe Laryngomalacia Requiring Supraglottoplasty. Arch Otolaryngol Head Neck Surg 2009;135:647-651

5) Thompson, DM. Abnormal sensorimotor integrative function of the larynx in congenital laryngomalacia: a new theory of etiology. Laryngoscope. 2007 Jun; 117: 1-33.

6) Yao TC, Chiu CY, Wu KC, Wu LJ, Huang JL. Failure to thrive caused by the coexistence of vallecular cyst, laryngomalacia and gastroesophageal reflux in an infant. Int J Pediatr Otorhinolaryngol 2004 Nov;68:1459-64.

7) Wong, KS, Li HY, and Huang, TS. Vallecular cyst synchronous with laryngomalacia: presentation of two cases. Otolaryngol Head Neck Surg 1995;113:621-624. 
Video 1:

Part 1 demonstrates the tongue base mass displacing the epiglottis posteriorly and contributing significantly to dynamic inspiratory collapse of the epiglottis. Note that the glottic inlet is barely visible.

Part 2 shows the supraglottic structures after decompression of the tongue base mass. Here, the severe degree of laryngomalacia is more readily apparent.

Part 3 demonstrates the excisional biopsy of the tongue base mass. 